\title{
LOW-DELAY PERFECT RECONSTRUCTION TWO-CHANNEL FIR/IIR FILTER BANKS AND WAVELET BASES WITH SOPOT COEFFICIENTS
}

\author{
W.Liu, S. C. Chan and K. L. Ho \\ Department of Electrical and Electronic Engineering \\ The University of Hong Kong, Pokfulam Road, Hong Kong.
}

\begin{abstract}
In this paper, a new family of two-channel low-delay filter banks and wavelet bases using the PR structure in [3] with SOPOT coefficients are proposed. In particular, the functions alpha( $z)$ and beta(z) in the structure are chosen as nonlinear-phase FIR and IIR filters, and the design of such multiplier-less filter banks is performed using the genetic algorithm. The proposed design method is very simple to use, and is sufficiently general to construct low-delay filter banks with flexible lengths, delays, and regularity. Several design examples are given to demonstrate the usefulness of the proposed method.
\end{abstract}

\section{INTRODUCTION}

Perfect reconstruction (PR) multirate filter banks have important applications in signal analysis, signal coding and the design of wavelet bases. Figure 1 shows the block diagram of an 2-channel maximally decimated filter banks. In [3], a new structure for two-channel perfect reconstruction FIR/IIR filter banks is proposed. It is parameterized by two functions $\alpha(z)$ and $\beta(z)$, which can be chosen as linear-phase FIR or all-pass functions to realize new classes of FIR and IIR filter banks with very low design and implementation complexities and good frequency responses. In [3], the case using identical $\alpha(z)$ and $\beta(z)$ with delay parameter $M=2 N-1$ (see Fig. 2) is studied to obtain linear-phase FIR or passband linear-phase IIR filter banks. Furthermore, by imposing the $K$-regularity condition, linear-phase FIR and passband linear-phase IIR wavelet bases can readily be obtained.

In this paper, we shall investigate the case in which $\alpha(z)$ and $\beta(z)$ are nonlinear-phase FIR or IIR functions. As the linearphase requirement is relaxed, the lengths of $\alpha(z)$ and $\beta(z)$ are no longer restricted by the delay parameters of the system. Therefore, higher stopband attenuation can still be achieved at low system delay. By representing each coefficient as sum of powers-of-two (SOPOT), multiplier-less filter banks and wavelet bases can be obtained. The design is performed using the genetic algorithm (GA). The proposed design method is very simple to use, and is sufficiently general to construct low-delay filter banks with flexible lengths, delays, and $K$-regularity condition for generating wavelet bases. Several design examples are given to demonstrate the usefulness of the proposed method. Design results show that GA is capable of finding very good filter banks and wavelets with very low implementation complexity. The average number of terms used per coefficient in the design examples ranges from 2.4 to 2.9. In other words, each coefficient multiplication can efficiently be implemented with 1.4 to 1.9 additions. The paper is organized as follows: Section II is a summary of the theory of twochannel filter banks and their related wavelet bases. The structure proposed in [3] and the basic idea behind the proposed low-delay filter banks and wavelet bases are also described. Section III is devoted to the proposed design method. Several examples will be given in section IV and conclusions are drawn in Section V.

\section{TWO-CHANNEL STRUCTURAL PR FILTER BANKS AND WAVELET BASES}

Fig. 1 shows the structure of a two-channel critically decimated filter banks. It can be shown that $[1,2]$ the reconstructed signal, $Y(z)$, is given by

$$
Y(z)=T(z) X(z)+A(z) X(-z)
$$

$$
\text { where } \quad \begin{aligned}
T(z) & =\frac{1}{2}\left[H_{0}(z) G_{0}(z)+H_{1}(z) G_{1}(z)\right], \\
A(z) & =\frac{1}{2}\left[H_{0}(-z) G_{0}(z)+H_{1}(-z) G_{1}(-z)\right] .
\end{aligned}
$$

The aliasing term, $A(z)$, can be canceled if the analysis and synthesis filters are chosen as follows

$$
G_{0}(z)=-H_{1}(-z), G_{1}(z)=H_{0}(-z) .
$$

Combining (2-1) and (2-2), one gets the following PR condition in $H_{0}(z)$ and $H_{1}(z)$

$$
T(z)=\frac{1}{2}\left[H_{0}(-z) H_{1}(z)-H_{0}(z) H_{1}(-z)\right]=c z^{-n_{0}}
$$

where $n_{0}$ is an integer and $c$ is a non-zero constant.

In [3], a new class of two-channel structurally-PR filter banks and wavelet bases are proposed (Fig.2). The expressions for the analysis filters are given by:

$$
H_{0}(z)=\frac{\left(z^{-2 N}+z^{-1} \beta\left(z^{2}\right)\right)}{2}, H_{1}(z)=-\alpha\left(z^{2}\right) H_{0}(z)+z^{-2 M-1} \text {. }
$$

It can be seen from (2-4) that (2-3) is satisfied for any choices of $\alpha(z)$ and $\beta(z)$. Therefore, FIR and IIR filter banks can readily be realized by choosing $\alpha(z)$ and $\beta(z)$ as polynomials or rational functions. In [3], the case of using identical $\alpha(z)$ and $\beta(z)$ is studied with the delay parameter $M$ chosen as $2 N-1$. New classes of FIR and IIR filter banks were obtained by choosing $\beta(z)$ and $\alpha(z)$ as Type-II linear-phase functions and all-pass functions, respectively. The design of $\beta(z)$ (and $\alpha(z)$ ) can be accomplished noting that $H_{0}(z)$ (and $\left.H_{1}(z)\right)$ will become ideal lowpass (and highpass) filters if $\beta(z)$ (and $\alpha(z)$ ) has the following magnitude and phase responses:

$$
\begin{aligned}
& \left|\beta\left(e^{j 2 m}\right)\right|=1 \quad \forall \omega \\
& \angle \beta\left(e^{j 2(\prime \prime)}\right)=\left\{\begin{array}{ccc}
(-2 N+1) \omega & \text { for } & \omega \in[0, \pi / 2] \\
(-2 N+1) \omega \pm \pi & \text { for } & \omega \in(\pi / 2, \pi]
\end{array}\right.
\end{aligned}
$$

Moreover, by imposing zeros at $\omega=\pi$ for $H_{0}(z)$ and $\omega=0$ for $H_{1}(z)$, wavelet bases can be constructed from the resulting filter banks. In fact, the theory of wavelet is closely related to that of multirate filter banks. It has been shown that discrete dyadic wavelets can be obtained from two-channel PR filter banks with added regularity condition [4,5]. For biorthogonal dyadic wavelet bases, $H_{0}(z)$ and $G_{0}(z)$ should have $K_{0}$ (or $\tilde{K}_{0}$ ) zeros at $z=-1$ (the $K$-regularity condition). In addition, $H_{1}(z)$ and $G_{1}(z)$ should also have at least one zero at $z=1[5]$ :

$$
\begin{aligned}
& \left.\frac{d^{k} H_{0}(z)}{d z^{k}}\right|_{:=-1}=0, k=0,1, \ldots, K_{0}-1 ;\left.\frac{d^{k} G_{0}(z)}{d z^{k}}\right|_{:=-1}=0, \\
& k=0,1, \ldots, \tilde{K}_{0}-1 . \\
& H_{1}(1)=G_{1}(1)=0 .
\end{aligned}
$$

The delay of the system proposed in [3] is:

$$
n_{0}=2 N+2 M+1 \text {. }
$$

If $\alpha(z)$ and $\beta(z)$ are identical and are chosen as Type-II linear-phase functions or all-pass functions, then the system delay 
will be predetermined by the length of $\alpha(z)$ or $\beta(z)$. The only way to reduce the system delay in some low-delay applications is to reduce the length of the filters, which will unavoidably reduce the stopband attenuation of the filter banks. To overcome this problem, nonlinear-phase FIR or IIR functions have to be used for $\alpha(z)$ and $\beta(z)$ so that more flexibility are available in choosing the filter length and hence their stopband attenuation. The system delay is still given by (2-8). But the lengths of $\alpha(z)$ and $\beta(z)$ are no longer limited by the values of $M$ and $N$. In $[14,15]$, methods for designing such low-delay FIR/IIR filter banks and wavelets using the Remez exchange algorithm and model reduction technique, are proposed. In this work, we consider their multiplier-less realization using the sum of powers-of-two coefficients representation.

\section{THE PROPOSED DESIGN METHODS}

As mentioned earlier, $\alpha(z)$ and $\beta(z)$ considered in this paper are nonlinear-phase. Since the filter banks are still PR if the coefficients of $\alpha(z)$ and $\beta(z)$ are quantized to other values, it is possible to reduce its complexity by expressing them in sum of powers-of-two coefficients. By so doing, each coefficient multiplication can be efficiently performed by simple additions and shifts. A number of methods have been proposed for designing FIR filters with SOPOT coefficients. A classical work is the integer programming method proposed by Lim [9]. Other heuristic methods such as stimulated annealing [10] and genetic algorithm [11] have also been proposed. In [7,12], the genetic algorithm (GA) is used to design SOPOT multiplier-less 2-channel orthogonal and biorthogonal linear-phase filter banks using respectively the structure in [2] and the transformation method.

In this paper, the GA is also employed to search for the SOPOT coefficients of $\alpha(z)$ and $\beta(z)$ in the proposed multiplierless low-delay filter banks and wavelet bases. Each coefficient of $\alpha(z)$ and $\beta(z)$ is represented as follows:

$$
h(n)=\sum_{k=1}^{p_{n}} a_{k} .2^{b_{k}}, \quad a_{k} \in\{-1,1\} b_{k} \in\{l, \ldots, 1,0,-1, \ldots,-l\} .
$$

" $l$ " is a positive integer and its value determines the range of coefficients. $p_{n}$ is the number of terms of the n-th coefficient. Normally, $p_{n}$ is limited to a small number, and the multiplication of such SOPOT coefficient can be implemented with simple shifts and additions. In this work, each coefficient is allowed to have different number of terms. is:

In the filter banks case, the objective function we minimize

$E\left(H_{k}\right)=\max \left\|H_{k}(\omega)|-| \tilde{H}_{k}(\omega)\right\|, k \in\{0,1\}, \omega \in[0, \pi],(3-2)$

where $\tilde{H}_{k}(\omega)$ is the desired frequency response of the $k$-th channel.

To obtain the corresponding wavelet bases, we need to incorporate the $K$-regularity conditions in (2-6) and (2-7). If we substitute (2-3) into (2-6) and (2-7), one gets a set of linear equations that have to be satisfied. The problem is a constrained nonlinear optimization, which cannot be easily handled by conventional genetic algorithm. Fortunately, it is found that these conditions can be used to eliminate some of the design variables, and it leads to an unconstrained optimization with fewer variables. From the GA point of view, the resulting problem is surprisingly easier to solve than that without the K-regularity constraints. In other words, it is easier to design the wavelet bases than to design the corresponding filterbanks, using GA.

Next, we give several examples to illustrate the proposed method.

\section{DESIGN EXAMPLES}

\section{Low-delay FIR filter banks.}

Both $\alpha(z)$ and $\beta(z)$ are low-delay nonlinear-phase FIR filters with length $L_{\alpha}$ and $L_{\beta}$, and with delay $D_{c \alpha}$ and $D_{\beta}$.

Example 4.1: The specifications are: $M=8, N=3$, cutoff frequencies: $\omega_{1}=0.4 \pi, \omega_{2}=0.6 \pi$, length: $L_{\alpha}=14, L_{\beta}=12$, delay: $D_{\alpha}=5.5, D_{\beta}=2.5$, overall system delay: $2 M+2 N+1=23$. The coefficients of the SOPOT filter banks and its frequency responses are summarized in Table 1 and Figure 3, respectively. The stopband attenuation is $40 \mathrm{~dB}$ and the average number of terms used per coefficient is 2.6.

\section{The low-delay FIR wavelet bases.}

To obtain the corresponding wavelet bases, now we incorporate the $K$-regularity conditions to the low-delay FIR filter banks. Let's express the coefficients of $\alpha(z)$ and $\beta(z)$ as $\alpha(0), \alpha(1) \ldots . .$. and $\beta(0), \beta(1) \ldots . . .$, respectively. Using $\left.\frac{d^{k} H_{0}(z)}{d z^{k}}\right|_{z=-1}=0$, we get for $k=0$ the following

$$
\left.\beta(z)\right|_{i=1}=1 \text { or equivalently } \sum_{i=0}^{L_{\theta}-1} \beta(i)=1 .
$$

And for $k=1,2, \ldots \ldots$

$$
\begin{aligned}
& (-2 N)(-2 N-1) \cdots(-2 N-k+1) z^{-2 N-k}+ \\
& \left.\sum_{i=0}^{L_{\beta}-1}(-2 i-1) \cdots(-2 i-k) \beta(i) z^{-2 i-1-k}\right|_{:=-1}=1
\end{aligned} .
$$

On the other, from $\left.\frac{d^{k} G_{0}(z)}{d z^{k}}\right|_{z=-1}=0$, we get $\left.\frac{d^{k} H_{1}(z)}{d z^{k}}\right|_{\varepsilon=1}=0$.

For $k=0,\left.\quad \alpha(z)\right|_{:=1}=1$, or $\sum_{i=0}^{t_{x}-1} \alpha(i)=1$.

and for $k=1$,

$$
-\alpha\left(z^{2}\right) H_{0}(z)-2 z \alpha\left(z^{2}\right) H_{0}(z)+\left.(-2 M-1) z^{-2 M-2}\right|_{z=1}=0 .
$$

Similar conditions can be got if we keep on differentiating the equation for $k=2,3, \ldots$

Example 4.2: The specifications are: $K_{0}=\tilde{K}_{0}=3, M=5, N=2$, $L_{\alpha}=10, L_{\beta}=8$; cutoff frequencies: $0.38 \pi, 0.62 \pi$; delays of $\alpha(z), \beta(z)$ and the system are $3.5,1.5$, and 15 , respectively. By solving the corresponding equations in (4-1), one gets :

$$
\begin{aligned}
\beta(2)= & (3-24 \beta(3)-48 \beta(4)-80 \beta(5) \\
& -120 \beta(6)-168 \beta(7)) / 8 \\
\beta(1)= & (3-4 \beta(2)-6 \beta(3)-8 \beta(4)-10 \beta(5) \\
& -12 \alpha(6)-14 \beta(7)) / 2 \\
\beta(0)= & 1-\beta(1)-\beta(2) \cdots \cdots-\beta(7) \\
\alpha(2)= & 35 / 8-3 \alpha(3)-6 \alpha(4)-10 \alpha(5)-15 \alpha(6) \\
& -21 \alpha(7)-28 \alpha(8)-36 \alpha(9) \\
\alpha(1)= & 7 / 2-2 \alpha(2)-3 \alpha(3)-4 \alpha(4) \cdots \cdots-9 \alpha(9) \\
\alpha(0)= & 1-\alpha(1)-\alpha(2) \cdots \cdots-\alpha(9) .
\end{aligned}
$$

By eliminating these variables, we only have $8+10-6=12$ free parameters to optimize, 6 less than the original filterbanks. The average number of terms used per coefficient is 2.9 and the stopband attenuation is $30 \mathrm{~dB}$. The coefficients of the SOPOT wavelet filter banks and its frequency responses are summarized in Table 2 and Figure 4, respectively.

\section{Low-Delay IIR Filter Banks.}

In general, both $\alpha(z)$ and $\beta(z)$ can be IIR filters. In the design process, however, we find that its performance/complexity tradeoff is not satisfactory. In fact, it is more efficient to choose one of the $\alpha(z)$ or $\beta(z)$ to be a nonlinear-phase FIR filter and the other as 
an IIR filter. Much better performance can be achieved with similar implementation complexity.

Example 4.3: In this example, $\beta(z)$ is an IIR filter with delay 1.5 and is given by $\beta(z)=\frac{\left(1+c_{0} z^{-1}+c_{1} z^{-2}+c_{2} z^{-3}\right) c_{6}}{\left(1+c_{3} z^{-1}+c_{4} z^{-2}+c_{5} z^{-3}\right)} . \alpha(z)$ is a nonlinear-phase FIR filter with length $L_{k}=11$ and delay 3.5 . The specifications are: $M=5, N=2$; cutoff frequencies: $\omega_{1}=0.4 \pi$, $\omega_{2}=0.6 \pi$; overall system delay: 15 . The stopband attenuation of the resulted filter banks is $33 \mathrm{~dB}$; and the average number of terms used per coefficient is 2.4. The coefficients of the SOPOT wavelet filter banks and its frequency responses are summarized in Table 3 and Figure 5, respectively.

\section{Low-Delay IIR Wavelet Bases.}

Example 4.4: In this example, we choose $\beta(z)$ as a FIR filter with length $L_{\beta}=8$ and delay 1.5 . The constraints are the same as equations (4-2a), (4-2b), and (4-2c). $\alpha(z)$ is an all-pass filter with order 3 and delay 2.5: $\alpha(z)=\frac{\sum_{k=0}^{3} a_{3-k} z^{-k}}{\sum_{k=0}^{3} a_{k} z^{-k}}$, which automatically fulfills the requirement $\left.\alpha(z)\right|_{i=1}=1$. Therefore, $K_{0}=3$. and $\tilde{K}_{0}=1$. The reason for the choice is to demonstrate the flexibility of the proposed design method using genetic algorithm. It will be seen that the performance of using the all-pass filter is very good and its complexity is extremely low. The specifications are: $M=4, N=2$; cutoff frequencies: $\omega_{1}=0.34 \pi, \omega_{2}=0.66 \pi$, overall system delay: 13 . The stopband attenuation of this example is $36 \mathrm{~dB}$ and the coefficients of the SOPOT wavelet filter banks and its frequency responses are summarized in Table 4 and Figure 6 , respectively. The average number of terms used per coefficient is 2.4 .

\section{CONCLUSION}

A new family of multiplier-less two-channel low-delay filter banks and wavelet bases using the PR structure in [3] are presented. The functions $\alpha(z)$ and $\beta(z)$ in the structure are chosen as nonlinear-phase FIR and IIR filters, and the design of such multiplier-less filter banks and wavelet bases is performed using the genetic algorithm. It was found that GA is able to find very good solution to this problem. The proposed design method is very simple to apply, and is sufficiently general to construct low-delay filterbanks with flexible lengths, delays, and number of zeroes at $\pi$ (or 0 ) in their analysis filters to generate the corresponding wavelet bases.

\section{REFERENCES}

[1] P. P. Vaidyanathan, Multirate systems and filter banks. Englewood Cliffs, NJ: Prentice Hall, 1992.

[2] C.Sidney Burrus, Ramesh A.Gopinath, and Haitao Guo. "Introduction to wavelets and wavelet transforms". Prentice Hall, 1998.

(3) S. M. Phoong, C. W. Kim and P. P. Vaidyanathan, "A new class of two-channel biothogonal filter banks and wavelet bases", IEEE Trans. SP., Vol.43, No. 3, pp.649-664, March 1995.

[4] I. Daubechies, "Orthonormal bases of compactly supported wavelets," Commun. Pure Appl. Math., Nov. 1988, Vol. 41, pp. 909-996

[5] A. Cohen, I. Daubechies and J. Feauveau, "Biorthogonal bases of compactly supported wavelets," Comm. Pure Applied. Math. Vol. 45, pp. 485-560, 1992.

[6] K.S. Tang, K.F.Man, S.Kwong and Q.He, "Genetic algorithms and their applications", IEEE Signal Processing Magazine, pp. 22-37, Nov 1996.
[7] S. Sriranganathan, D. R. Bull, D. W. Redmill, "The design of Low Complexity Two-Channel Lattice-Structure PerfectReconstruction Filter Banks Using Genetic Algorithm". Proc. of ISCAS '97., vol.4, pp.2393-2396, June 1997.

[8] Al-Adnani, A.; Chapman, R.; Durrani, T.S., "Time-domain design of low delay wavelet transforms", Electronics Letters, Vol. 29. No. 9, pp. 2177 - 217, Dec. 1993

[9] Y. C. Lim, S. R. Parker, "FIR filter design over a discrete power-of-two coefficients space," IEEE Trans. Acoust. Speech, Signal Processing, vol. ASSP-31, pp. 583-591, June 1983.

[10] N. Benvenuto, M. Marchesi, A. Uncini, "Applications of simulated annealing for the design of special digital filters," IEEE Trans. on Signal Processing, Vol. 40, No. 2, pp. $33-$ 332, Feb. 1992

[11] D. W. Redmill and D. R. Bull, "Automated design of low complexity FIR filters," Proc. IEEE ISCAS'98, vol. 5, pp. 429-432, 1998.

[12] D. W. Redmill, D. R. Bull, and R. R. Martin, "Design of multiplier free linear phase perfect reconstruction filter banks using transformations and genetic algorithms, Sixth International Conference on Image Processing and Its Applications, Vol. 2, pp. 766-770, 1997.

[13] X. Zhang and T. Yoshikawa, "Design of Two-Channel Stable IIR Perfect Reconstruction Filter Banks," IEICE Trans. Fundamentals, Vol. E81-A, No.8, pp. 1592-1597, August 1998.

[14] J. S. Mao, S. C. Chan, W. Liu, and K. L. Ho, "Design and multiplier-less implementation of a class of two-channel PR FIR filter banks and wavelets with low system delay," submitted for publication, Oct., 1999.

[15] S. C. Chan, J. S. Mao, and K. L. Ho, "Design of two-channel perfect reconstruction IIR filter banks with low system delay using model reduction, " under preparation.

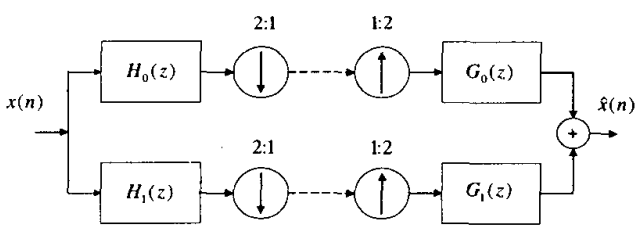

Fig. 1. Two-channel multirate filter bank.
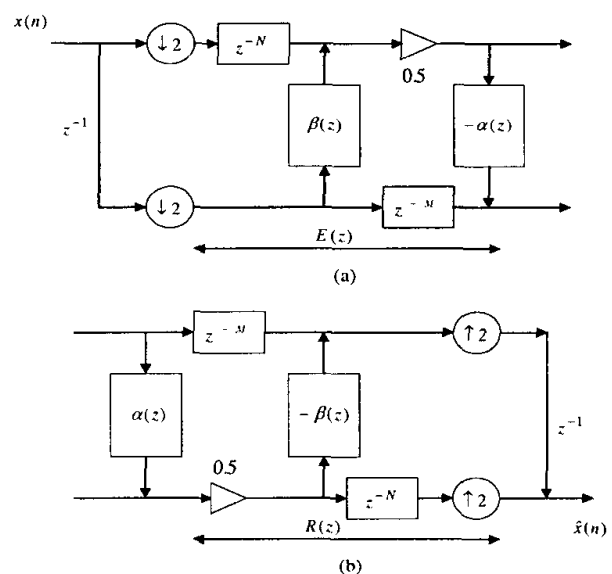

Fig. 2. Two-channel PR filter bank proposed in [3]: (a) analysis filter, (b) synthesis filter. 
$\mathrm{dB}$

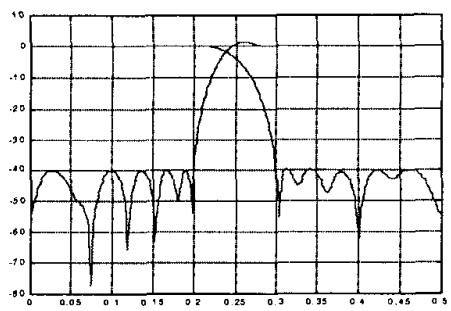

Fig. 3. Frequency responses of analysis filters of Example 4.1.

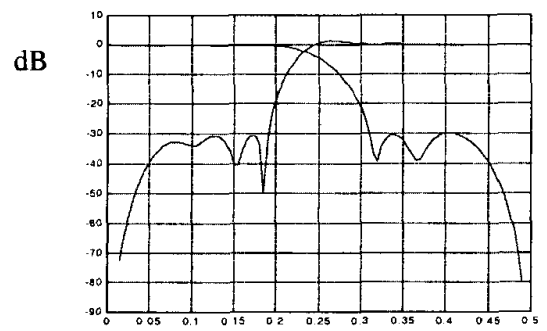

(a)
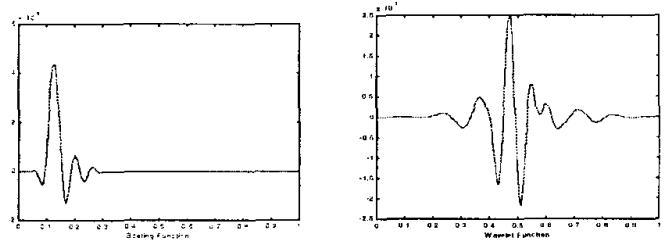

(b)

Fig. 4. Wavelet filter banks in Example 4.2 (a) Frequency responses of analysis filters, (b) scaling and wavelet functions ( $K=3, \tilde{K}=3$ ).

dB

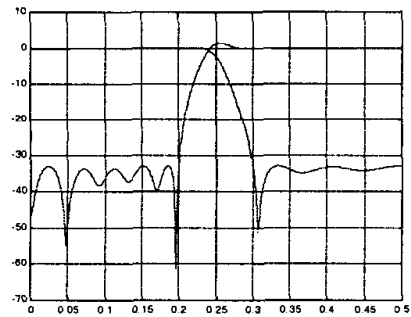

Fig. 5. Frequency responses of analysis filters of Example 4.3 .

$\mathrm{dB}$

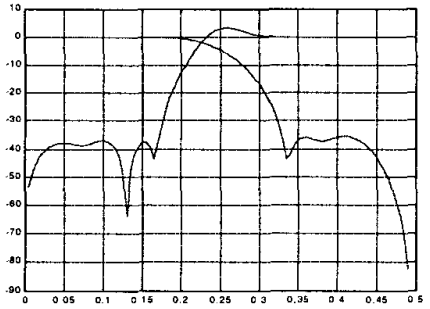

(a)
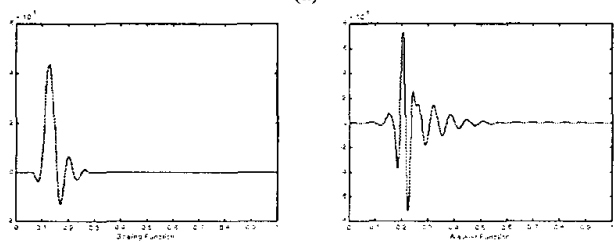

(b)
Fig. 6. Wavelet filter banks in Example 4.4. (a) Frequency responses of analysis filters, (b) scaling and wavelet functions $(K=3, \tilde{K}=1)$.

\begin{tabular}{|c|c|c|}
\hline 0 & $-2^{-8}$ & $2^{-5}+2^{-7}+2^{-8}$ \\
\hline 1 & $2^{-6}+2^{-8}+2^{-10}$ & $-2^{-3}+2^{-8}$ \\
\hline 2 & $-2^{-5}-2^{-6}$ & $2^{-1}+2^{-4}-2^{-6}$ \\
\hline 3 & $2^{-4}+2^{-6}+2^{-9}$ & $2^{-1}+2^{-2}-2^{-5}$ \\
\hline 4 & $-2^{-2}+2^{-4}+2^{-6}$ & $-2^{-2}-2^{-5}-2^{-7}$ \\
\hline 5 & $2^{-1}+2^{-3}-2^{-6}$ & $2^{-2}-2^{-4}-2^{-8}$ \\
\hline 6 & $2^{-1}+2^{-3}+2^{-5}$ & $-2^{-3}-2^{-7}$ \\
\hline 7 & $-2^{-2}+2^{-5}+2^{-8}$ & $2^{-3}-2^{-5}$ \\
\hline 8 & $2^{-3}-2^{-8}$ & $-2^{-4}-2^{-10}$ \\
\hline 9 & $-2^{-4}-2^{-7}-2^{-8}$ & $2^{-5}+2^{-7}-2^{-10}$ \\
\hline 10 & $2^{-4}-2^{-6}-2^{-9}$ & $-2^{-6}-2^{-7}-2^{-10}$ \\
\hline 11 & $-2^{-5}+2^{-8}$ & $2^{-7}+2^{-8}-2^{-10}$ \\
\hline 12 & $2^{-6}-2^{-10}$ & \\
\hline 13 & $-2^{-7}$ & \\
\hline
\end{tabular}

Table 1.

\begin{tabular}{|c|c|c|}
\hline$i$ & $\alpha(i)$ & $\beta(i)$ \\
\hline 0 & $-2^{-6}-2^{-8}$ & $-2^{-4}-2^{-6}-2^{-9}$ \\
\hline 1 & $2^{-3}-2^{-5}-2^{-7}-2^{-9}$ & $2^{-1}+2^{-5}+2^{-7}+2^{-9}$ \\
\hline 2 & $-2^{-3}-2^{5}-2^{-6}-2^{-4}$ & $2^{-4}+2^{-2}-2^{-6}-2^{-9}$ \\
\hline 3 & $2^{-1}+2^{4}+2^{5}$ & $-2^{-2}-2^{-5}-2^{-7}-2^{-9}$ \\
\hline 4 & $2^{-1}+2^{-3}+2^{-6}$ & $2^{-3}+2^{-5}$ \\
\hline 5 & $-2^{-3}-2^{-4}-2^{-7}$ & $-2^{-3}+2^{-6}+2^{-8}$ \\
\hline 6 & $2^{-3}-2^{-6}$ & $2^{-4}-2^{-8}$ \\
\hline 7 & $-2^{-4}+2^{-6}-2^{-8}$ & $-2^{-6}+2^{-8}$ \\
\hline 8 & $2^{-6}+2^{-7}$ & \\
\hline 9 & $-2^{-7}-2^{-9}$ & \\
\hline
\end{tabular}

Table 2.

\begin{tabular}{|l|l|l|}
\hline$i$ & $\alpha(i)$ & $c_{i}$ \\
\hline 0 & $-2^{-5}-2^{-7}$ & $-2^{2}+2^{-1}-2^{-5}$ \\
\hline 1 & $2^{-4}+2^{-5}-2^{-9}$ & $-2^{3}-2^{0}+2^{-3}$ \\
\hline 2 & $-2^{-3}-2^{-5}$ & $-2^{0}-2^{-1}-2^{-4}$ \\
\hline 3 & $2^{-1}+2^{-9}-2^{-6}-2^{-7}$ & $2^{-1}+2^{-3}+2^{-4}+2^{-8}$ \\
\hline 4 & $2^{-1}+2^{-3}+2^{-5}+2^{-9}$ & $-2^{-7}$ \\
\hline 5 & $-2^{-2}+2^{-5}$ & $2^{-6}$ \\
\hline 6 & $2^{-3}-2^{-8}$ & $-2^{-3}$ \\
\hline 7 & $-2^{-4}-2^{-7}$ & \\
\hline 8 & $2^{-5}+2^{-6}$ & \\
\hline 9 & $-2^{-5}+2^{-7}+2^{-10}$ & \\
\hline 10 & $2^{-6}$ & \\
\hline
\end{tabular}

Table 3

\begin{tabular}{|c|c|c|}
\hline$i$ & $\beta(i)$ & $a_{i}$ \\
\hline 0 & $-2^{-4}-2^{-7}+2^{-9}$ & $2^{0}$ \\
\hline 1 & $2^{-1}+2^{-6}+2^{-9}$ & $2^{-1}-2^{-5}+2^{-9}$ \\
\hline 2 & $2^{-1}+2^{-2}-2^{-4}+2^{-8}$ & $-2^{-4}-2^{-5}+2^{-9}$ \\
\hline 3 & $-2^{-2}-2^{-5}$ & $2^{-5}-2^{-9}$ \\
\hline 4 & $2^{-3}+2^{-6}$ & \\
\hline 5 & $-2^{-4}-2^{-7}$ & \\
\hline 6 & $2^{-5}-2^{-7}+2^{-9}$ & \\
\hline 7 & $-2^{-9}$ & \\
\hline
\end{tabular}

Table 4. 